\title{
Predictive Current Control Applied to a 3L-NPC Inverter
}

\author{
Diego Rojas, Marco Rivera, Javier Muñoz, Carlos Baier, Patrick Wheeler,
}

\begin{abstract}
In recent years the application of Model Based Predictive Control (MPC) for power converters has been successfully presented as an alternative to traditional control techniques. MPC has attracted interest due to its fast dynamic response and the ability to incorporate various control requirements in a cost function. This work describes the fundamental aspects of the MPC, from its principle of operation to its application to control in multilevel power converters. The application of MPC technique for the control of a three-level neutral point clamped inverter (3L-NPC) will be analysed for current control in a three-phase load, the balance in the capacitors link DC, and the associated dynamic response. The MPC method is analysed and simulation results from a Matlab/Simulink model with different sampling times are presented.
\end{abstract}

Keywords-Power converter, predictive control, three level inverter.

\section{INTRODUCCIÓN}

$\mathbf{E}$ L Control Predictivo Basado en Modelo (MPC) se define como una una técnica de control que utiliza la forma explícita del modelo matemático del proceso a ser controlado, conocido como modelo de predicción. Este modelo, es utilizado para determinar el comportamiento futuro de las variables a ser controladas a lo largo de un intervalo de tiempo futuro, que se define como horizonte temporal de predicción. De esta forma es posible calcular las variables de control futuras, de tal forma de lograr que las variables controladas converjan a ciertos valores de referencias en dicho horizonte. Pero hay que tener en cuenta que como es una técnica de control de optimización que se basa en el modelo del sistema, implica una gran cantidad de cálculos [1].

Gracias a los avances tecnológicos y al surgimiento de microcontroladores más rápidos, que son capaces de realizar cálculos más potentes, el control predictivo se ha convertido en un método de control alternativo para aplicaciones de convertidores de potencia. Esta técnica es un concepto muy intuitivo

Diego Rojas. Estudiante del Doctorado en Sistemas de Ingeniería, Facultad de Ingeniería, Universidad de Talca, Curicó, Chile, (e-mail: diego.rojaso@utalca.cl).

Marco Rivera. Director del Laboratorio de Conversión de Energías y Electrónica de Potencia, Universidad de Talca, Curicó, Chile, (e-mail: marcoriv@utalca.cl).

Javier Muñoz. Departamento de Ingeniería Eléctrica, Facultad de Ingeniería, Universidad de Talca, Chile (email: jamunoz@utalca.cl).

Carlos Baier. Departamento de Ingeniería Eléctrica, Facultad de Ingeniería, Universidad de Talca, Chile (email: cbaier@utalca.cl).

Patrick Wheeler. Power Electronics, Machines and Control Group, The University of Nottingham, Nottingham, UK, (e-mail: Pat.Wheeler@nottingham.ac.uk).

978-0-7381-3333-1/21\$31.00 @2021 IEEE que es fácil de implementar y funciona bien considerando numerosas restricciones [2].

Los convertidores multinivel con diodo de enclavamiento generalmente se consideran para escenarios en media tensión y alta potencia [3]. De hecho, pueden usarse a altos niveles de potencia con voltajes de modo común reducidos, distorsión armónica total e interferencia electromagnética [4]. Estos convertidores emplean diodos de sujeción y condensadores de DC en cascada para producir formas de onda de voltaje de AC con múltiples niveles. Los convertidores de tres niveles con diodo de enclavamiento a menudo se conocen como los convertidores de punto neutro (NPC) y se utilizan en muchas aplicaciones industriales.

A continuación, se citan las ventajas y desventajas más importantes de los inversores multinivel [5]:

- Cuanto mayor sea la cantidad de niveles diseñado, menor es la tensión soportada por los interruptores de potencia.

- Los diodos de enclavamiento logran fijar las escalas de voltaje en la salida del inversor y pueden obtenerse elevadas tensiones es por eso que deben ser conectados en serie.

- Con la carga y descarga de los condensadores del bus de DC, es posible controlar el flujo de potencia, es por eso que deben ser compensados de manera a mantener el punto neutro.

- Al aumentar el número de niveles, aumenta la complejidad de construcción y los costos de implementación.

- Para lograr el correcto enclavamiento de las tensiones de bloqueo sobre los interruptores de potencia se debe realizar conmutaciones secuenciales entre niveles sucesivos, para lo cual se debe de tener una regla sobre la selección de los estados de conmutación.

En este trabajo, se analizará la implementación de la técnica MPC a un inversor de punto neutro fuente de tensión de tres niveles (3L-NPC). El método de control MPC se analiza y simula teóricamente en Matlab/Simulink con tiempos de muestreos distintos.

\section{Control Predictivo Basado en Modelo}

El control de los convertidores de potencia ha sido ampliamente estudiados en las literaturas donde se presentan nuevos esquemas de control cada año. Se han propuesto varios esquemas de control aplicados a los convertidores de potencia [6], entre ellos, el MPC presenta varias ventajas [7], que lo hacen adecuado para el manejo de la energía en los convertidores de potencia, entre las cuales están:

- Es un concepto muy intuitivo y fácil de entender. 
- Es posible aplicarlo a una gran variedad de sistemas.

- El caso multivariable puede ser fácilmente considerado.

- Es fácil de incluir no linealidades y restricciones.

- Respuesta dinámica rápida.

- Es posible compensar los tiempos muertos.

- El controlador resultante es fácil de implementar.

- La metodología es abierta de manera a incluir modificaciones y extensiones dependiendo de las especificaciones.

Pero presentan desventajas como:

- La calidad del controlador depende de la calidad del modelo.

- Gran cantidad de cálculos comparado con los métodos clásicos.

La técnica de control MPC consiste en obtener el comportamiento futuro de las variables a ser controladas, para ello hace uso del modelo que define la dinámica del sistema. Esta predicción es utilizada por el controlador de manera a obtener una actuación óptima según lo planteado como objetivo de control [8]. De este modo, los pasos para el diseño de la técnica de control MPC son:

1) El convertidor se modela como un sistema de estados finitos, que identifica todos los estados de conmutación posible.

2) Un modelo de la carga es utilizado para predecir el comportamiento de cada estado posible.

3) Una función de costo $(\mathrm{CF})$ es definida, la cual representa el comportamiento deseado del sistema.

4) Se elige el estado con que debe conmutar el convertidor de potencia con el fin de minimizar la CF.

El modelo de tiempo discreto es utilizado para la predicción y se puede expresar como un modelo en espacios de estados de la siguiente manera:

$$
\begin{gathered}
x(k+1)=A x(k)+B u(k) \\
y(k)=C x(k)+D u(k)
\end{gathered}
$$

Es necesario definir una función de costo que represente el comportamiento deseado del sistema. Esta función tiene en cuenta las referencias, los estados y las actuaciones futuras.

$$
J=f(x(k), u(k), \ldots, u(k+N))
$$

El MPC se define como un planteamiento de optimización que consiste en obtener el menor valor posible de la CF $J$, para un horizonte predefinido en el tiempo $N$, sujeto al modelo del sistema y las restricciones impuestas. El resultado es una secuencia de $N$ actuaciones óptimas. El controlador consiste en aplicar solo el primer elemento de la secuencia:

$$
u(k)=\left[\begin{array}{llll}
1 & 0 \ldots 0
\end{array}\right] \arg \min _{u} J
$$

esto se realiza para cada instante de muestreo, utilizando los nuevos datos medidos, y de esa forma se obtienen nuevas secuencias de actuación óptimas.

El principio de funcionamiento del MPC se resume en la Fig. 1, los valores futuros de los estados del sistema se pronostican en un horizonte predefinido en el tiempo $k+N$ utilizando el modelo del sistema y la información que se dispone (medidas) hasta el tiempo $k$. La secuencia de las actuaciones óptimas se calcula gracias a la reducción de la $\mathrm{CF}$ y se aplica el primer elemento de la secuencia. Este esquema se vuelve a repetir de nuevo para cada instante de muestreo, considerando los nuevos datos medidos [9].

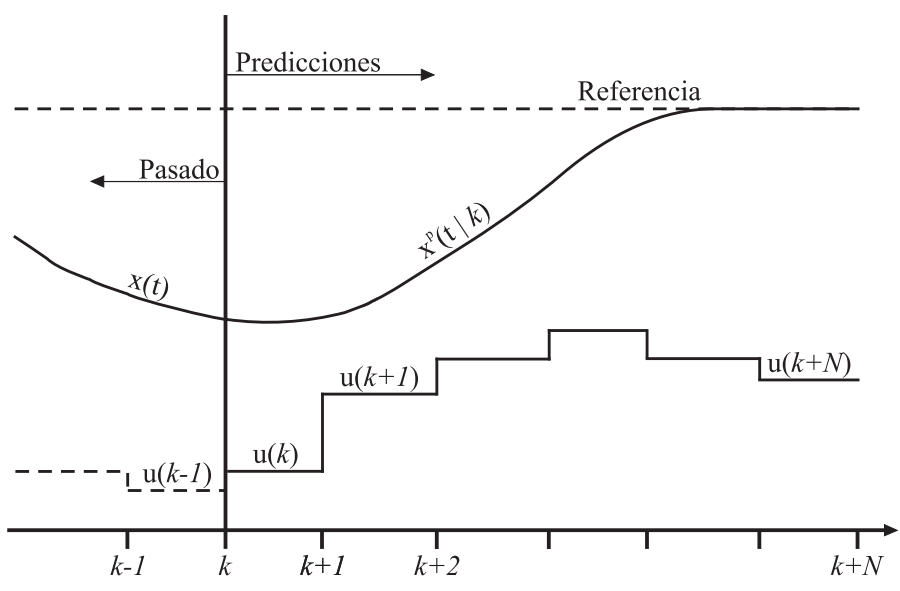

Fig. 1. Principio de operación de la técnica MPC.

\section{A. Modelo de los Convertidores de Potencia}

El modelo de los convertidores de potencia consiste en un conjunto de interruptores de potencia que actúan en dos estados, corte y saturación [10], esta condición es representado en la ecuación (1), de esta forma se tiene una cantidad finita de posibles maneras de combinar los estados de conmutación.

$$
S_{x}= \begin{cases}0 & \text { interruptor } S_{x} \text { desactivado } \\ 1 & \text { interruptor } S_{x} \text { activado }\end{cases}
$$

Donde $S_{x}$ representa el estado de conmutación de los interruptores.

\section{B. Objetivos de Control}

Los objetivos de control en sistemas basados con convertidores de potencia son: seguir referencias de corriente, voltaje, potencia, etc. Estos objetivos son representados en el MPC mediante la CF que pueden expresarse de forma general como en la ecuación (2), la cual calcula el error de la señal de referencia definida (valores esperados) con las señales predichas de las variables a controlar:

$$
g=\left\|x^{*}-x^{p}\right\|
$$

$x^{*}$ representa el valor de referencia de la variable a controlar y $x^{p}$ representa el valor predicho de la variable a controlar. La norma $\|$.$\| es una medida de la distancia entre la referencia$ y el valor predicho, por lo general, también es posible aplicar como valor absoluto, un valor cuadrado o un valor integral del error para un período de muestreo [11]:

$$
\begin{gathered}
g=\left|x^{*}-x^{p}\right| \\
g=\left(x^{*}-x^{p}\right)^{2}
\end{gathered}
$$




$$
g=\left|\int_{k}^{k+1}\left(x^{*}(t)-x^{p}(t)\right) d t\right|
$$

\section{Control de un Inversor de Punto Neutro TRIFÁSICO.}

La forma de representar un inversor NPC incluyendo la etapa de DC-link se observa en la Fig. 2 [12]. Cada fase del inversor está compuesta por cuatro interruptores de potencia y dos diodos en antiparalelo. Los pares superiores e inferiores están enclavados al punto neutro por medio de diodos de conducción, que son referidos como diodos de enclavamiento, donde los dos interruptores centrales y los diodos permiten conectar la terminal de salida al punto neutral $N$ del enlace DC [13]. La etapa del DC-link este compuesto de dos con-



Fig. 2. Representación equivalente de un inversor NPC trifásico.

densadores similares $C_{d c}$ formando entre sí el punto nuetro $N$. El voltaje total en un instante en el DC-link está dado por la ecuación (6).

$$
v_{d c}=v_{d c, u p}+v_{d c, l o}
$$

donde $v_{d c, u p}$ y $v_{d c, l o}$ son los valores de voltajes que cae sobre los condensadores de arriba y abajo respectivamente.

El potencial en el punto neutro $N$ está dado por la ecuación (7):

$$
v_{N}=\frac{1}{2}\left(v_{d c, l o}-v_{d c, u p}\right)
$$

\section{A. Estados Conmutados y Voltajes}

Siendo $u_{x}$ la variable que representa el estado en que conmutan cada una de las fases $x \in\{a, b, c\}$, y puede tomar valores entre $\{-1,0,1\}$. Para cada fase, el inversor puede dar tres niveles distintos de voltajes. Los niveles de voltajes, definidos respecto al punto neutro $N$ del DC-link está dado por la ecuación (8).

$$
v_{x}= \begin{cases}v_{d c, u p} & \text { si } u_{x}=1 \\ 0 & \text { si } u_{x}=0 \\ -v_{d c, l o} & \text { si } u_{x}=-1\end{cases}
$$

Tabla I

VALORES ENTRE EL ESTADO CONMUTADO $u_{x}$, VOLTAJE DE FASE $v_{x}$ Y LOS ESTADOS DE LOS INTERRUPTORES $S_{x 1}-S_{x 4}$ PARA LA FASE $x$ DONDE $x \in$ $a, b, c$.

\begin{tabular}{cccccc}
\hline $\begin{array}{c}\text { Estado } \\
\text { conmutado }\end{array}$ & \multicolumn{4}{c}{$\begin{array}{c}\text { Estados de los } \\
\text { interruptores }\end{array}$} & $\begin{array}{c}\text { Voltaje } \\
\text { de fase }\end{array}$ \\
$u_{x}$ & $S_{x 1}$ & $S_{x 2}$ & $S_{x 3}$ & $S_{x 4}$ & $v_{x}$ \\
\hline 1 & 1 & 1 & 0 & 0 & $v_{d c, u p}$ \\
0 & 0 & 1 & 1 & 0 & 0 \\
-1 & 0 & 0 & 1 & 1 & $-v_{d c, l o}$ \\
\hline
\end{tabular}

En la Tabla I se puede observar de una manera resumida y ordenada la correspondencia entre los estados y voltajes para cada fase. Además, si se ignora como fluctúa el potencial en el punto neutro, la ecuación (8) puede ser aproximado por la ecuación (9). Es decir, la variable de estado de conmutación $S_{x}$ representa el estado de conmutación de la fase $x$, con $x \in\{a, b, c\}$ y tienen tres valores posibles indicados por $1,0,-1$, que representan las combinaciones de conmutación que generan $\frac{v_{d c}}{2}, 0 \mathrm{y}-\frac{v_{d c}}{2}$, respectivamente, en la salida del inversor.

$$
v_{x} \approx \frac{v_{d c}}{2} u_{x}
$$

La tensión en las tres fases se define como $v_{a b c}=\left[\begin{array}{lll}v_{a} & v_{b} & v_{c}\end{array}\right]^{T}$. Cada fase queda representada por $x$ y sea $S_{x 1}-S_{x 4}$ los cuatro interruptores de potencia, con $S_{x 1}$ el interruptor superior y $S_{x 4}$ el inferior. Como se observa en la Tabla I, los cuatro interruptores activos tienen un comportamiento de manera dual en cada una de las fases. El estado $u_{x}=1$, por ejemplo, hace referencia a los interruptores $S_{x 1}$ y $S_{x 2}$ activos y los interruptores inferiores $S_{x 3}$ y $S_{x 4}$ apagados. Entonces, existen $27\left(3^{3}\right)$ vectores desiguales de la forma $u_{a b c}=\left[\begin{array}{lll}u_{a} & u_{b} & u_{c}\end{array}\right]^{T}$. Aplicando la transformada en $\alpha-\beta$, los vectores $u_{\alpha \beta}=\left[u_{\alpha} u_{\beta}\right]^{T}$ son definidos como vectores de tensión, y $u_{a b c}$ son los estados de conmutación trifásica [14]. En la Fig. 3 se observa los vectores de tensión.

\section{B. Recorrido de la Corriente}

$\mathrm{Si}$ se tiene en cuenta una de las tres fases en el estado conmutado $u_{x}$. Se considera la corriente de fase $i_{x}$ positiva, entonces, el sentido de $i_{x}$ es del inversor a la carga como se puede apreciar en la Fig. 4, entonces si:

- $u_{x}=1$, se tiene que los interruptores superiores están activos mientras tanto los interruptores inferiores están apagados. La corriente de fase positiva circula desde la terminal superior del DC-link por los dos interruptores activos hasta la terminal de fase, como se muestra en la Fig. 4(a).

- $u_{x}=0$, se tiene que los dos interruptores del centro están activos, y el interruptor superior y el inferior están apagados. La corriente positiva circula desde el punto $\mathrm{N}$ a través del diodo de enclavamiento superior y el interruptor central positivo hasta la terminal de fase, como puede se muestra en la Fig. 4(b). 


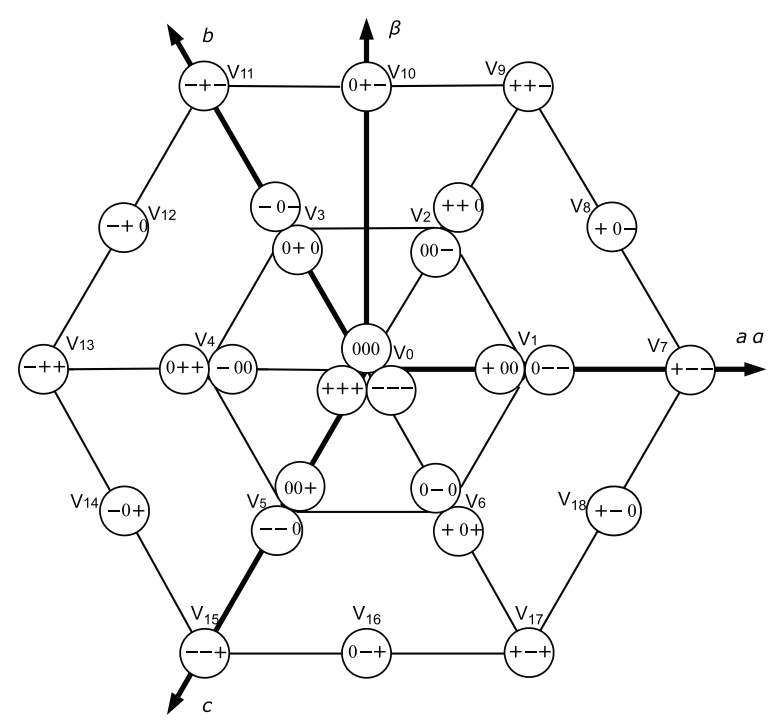

Fig. 3. Vectores posible de tensión y estados de conmutación generados por un inversor de tres niveles. Fuente: [15].

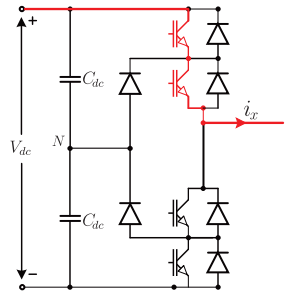

(a)

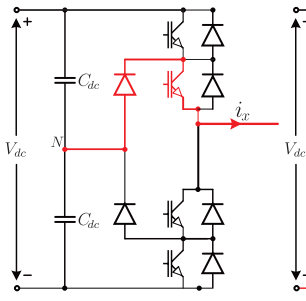

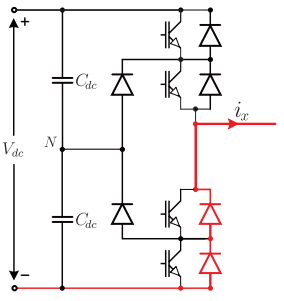

Fig. 4. Recorrido de la corriente en una fase del NPC, para una corriente de fase positiva.

- $u_{x}=-1$, se tiene que los dos interruptores inferiores están activos mientras que los superiores están apagados. La corriente positiva circula desde el terminal inferior del DC-link por los diodos en antiparalelo de los interruptores inferiores hasta la terminal de fase, como se muestra en la Fig. 4(c).

De manera análoga se puede derivar las corrientes de fase negativa.

\section{Predicción de la Corriente}

De manera a predecir las trayectorias futuras de las corrientes, el esquema de control predictivo requiere un modelo de la dinámica del sistema. La dinámica queda definida al obtener la ecuación de la tensión que cae sobre la carga RL, obtenida por la ley de tensión de Kirchhoff que se expresa en la ecuación (10).

$$
v(t)=R i(t)+L \frac{d i(t)}{d t}
$$

donde $v$ es la tensión de salida en el inversor, $R$ y $L$ son la resistencia y la inductancia de carga, respectivamente.

De manera a poder predecir el valor de la corriente futura en la carga se utiliza la forma discreta de la ecuación (10) para un tiempo de muestreo $T_{s}$.
Obteniendo una aproximación de la derivada $\frac{d i(t)}{d t}$ por:

$$
\frac{d i(t)}{d t}=\frac{i(k+1)-i(k)}{T_{s}}
$$

y reemplazando en la ecuación (10), se tiene la ecuación que describe la predicción de la corriente en la carga:

$$
i(k+1)=\left(1-\frac{R T_{s}}{L}\right) i(k)+\frac{T_{s}}{L} v(k)
$$

donde $v$ se calcula por la ecuación (9).

La forma en que varía los voltajes de los condensadores del enlace DC se describe mediante las ecuaciones diferenciales de los condensadores.

$$
\begin{aligned}
\frac{d v_{d c, u p}}{d t} & =\frac{1}{C_{d c}} i_{u p} \\
\frac{d v_{d c, l o}}{d t} & =\frac{1}{C_{d c}} i_{l o}
\end{aligned}
$$

donde $C_{d c}$ es el valor del capacitor. La misma aproximación de la derivada considerada anteriormente:

$$
\frac{d v_{d c, x}(t)}{d t}=\frac{v_{d c, x}(k+1)-v_{d c, x}(k)}{T_{s}}
$$

obteniendo las siguientes ecuaciones en tiempo discreto:

$$
\begin{gathered}
v_{d c, u p}(k+1)=v_{d c, u p}(k)+\frac{T_{s}}{C_{d c}} i_{u p}(k) \\
v_{d c, l o}(k+1)=v_{d c, l o}(k)+\frac{T_{s}}{C_{d c}} i_{l o}(k)
\end{gathered}
$$

Las corrientes $i_{u p}(k)$ y $i_{l o}(k)$ dependen del estado de conmutación del inversor y del valor de las corrientes de salida y se pueden calcular utilizando la siguiente expresión:

$$
\begin{aligned}
& i_{u p}(k)=i_{d c}(k)-H_{1 a} i_{a}(k)-H_{1 b} i_{b}(k)-H_{1 c} i_{c}(k) \\
& i_{l o}(k)=i_{d c}(k)-H_{2 a} i_{a}(k)-H_{2 b} i_{b}(k)-H_{2 c} i_{c}(k)
\end{aligned}
$$

donde $i_{d c}$ es la corriente proporcionada por la fuente de tensión $v_{d c}$. Las variables $H_{1 x}$ y $H_{2 x}$ dependen de los estados de conmutación y se definen como:

$$
\begin{aligned}
& H_{1 x}= \begin{cases}1 & \text { si } S_{x}=1 \\
0 & \text { para otro valor de } S_{x}\end{cases} \\
& H_{2 x}= \begin{cases}1 & \text { si } S_{x}=-1 \\
0 & \text { para otro valor de } S_{x}\end{cases}
\end{aligned}
$$

con $x \in a, b, c$. Por lo tanto, las ecuaciones (16) al (21) permiten predecir el efecto de seleccionar un estado de conmutación dado en la variación de las tensiones en los condensadores. 




Fig. 5. MPC para un inversor trifásico NPC.

\section{MPC de Corriente}

El diagrama de bloques del MCP aplicado al inversor NPC se muestra en la Fig. 5. El valor futuro de las corrientes se predice para los 27 estados de conmutación proporcionados por el inversor, mediante las ecuaciones (12), (16) y (17). Para este propósito, es necesario que mida la corriente de carga actual y el voltaje en los condensadores. Después de obtener las predicciones, se evalúa una CF ( $g$ ) para cada estado en que puede conmutar de forma segura el inversor. El estado de conmutación que reducer el valor de la CF se selecciona y aplica durante el siguiente periodo de muestreo.

Los requisitos de control para el inversor NPC son:

- Carga de seguimiento de referencia actual.

- Equilibrio de tensión de los condensadores del DC-link.

- Reducir la frecuencia con que conmutan los interruptores de potencia.

Estos requisitos se pueden formular a partir de una función de costo para minimizarla. La función de costo para el inversor NPC es el siguiente:

$$
g=\left|i_{\alpha}^{*}-i_{\alpha}^{p}\right|+\left|i_{\beta}^{*}-i_{\beta}^{p}\right|+\lambda_{d c}\left|v_{d c, u p}^{p}-v_{d c, l o}^{p}\right|+\lambda_{n} n_{c}
$$

Los dos primeros términos son los errores de corriente de carga en coordenadas $\alpha-\beta$, donde $i_{\alpha}^{p}$ e $i_{\beta}^{p}$ son las componentes reales e imaginarios del vector actual predicho, respectivamente, donde $i_{\alpha}^{*}$ y $i_{\beta}^{*}$ son las componentes real e imaginaria del vector actual de referencia, como se definió anteriormente. El tercer término en la CF calcula la resta de los valores predichos de los voltajes de los condensadores del enlace DC. Estos voltajes predichos se calculan utilizando las ecuaciones (16) y (17). Luego, al minimizar este término, los voltajes de los condensadores tenderán a ser iguales.

El último término es el número de conmutaciones requeridas para el estado de conmutación que implica menos conmutaciones de los semiconductores de potencia. De esta manera, el uso de este término tendrá un efecto directo en la frecuencia de conmutación y las pérdidas.

Los factores de ponderación $\lambda_{d c}$ y $\lambda_{n}$ manejan la relación entre los términos dedicados al seguimiento de referencia, el balance de voltaje y la disminución de la frecuencia de conmutación dentro de la función de costo $(g)$. Un gran valor de cierto $\lambda$ implica mayor prioridad para ese objetivo.

La frecuencia de conmutación promedio por semiconductor $f_{s}$ se definirá como el valor promedio de las frecuencias de conmutación de los 12 semiconductores de potencia controlada en el circuito convertidor. Así:

$$
f_{s}=\sum_{i=1}^{4} \frac{f_{\text {sai }}+f_{s b i}+f_{s c i}}{12}
$$

donde $f_{s k i}$ es la frecuencia de conmutación promedio durante un intervalo de tiempo del número de semiconductor de potencia (i) de la fase $(k)$, con $(i) \in\{1,2,3,4\}$ y $(k) \in\{a, b, c\}$. Como puede observar el lector, $f_{s}$ se definió como un promedio entre las frecuencias de conmutación. No todos los 12 semiconductores de potencia presentarán la misma frecuencia con la que conmuta.

\section{E. Implementación}

El Algoritmo 1 resume la manera de implementación del MPC para el control de corriente al 3L-VSI-NPC, donde $S(k)$ es el vector cuyos componentes contienen la información de la conmutación permitida de los interruptores.



\section{Resultados}

Los resultados de la simulación al aplicar el MPC al 3L-NPC en Matlab/Simulink se presentan tanto en estado estacionario como transitorio. Los parámetros de simulación son, fuente de tensión $V=1910.5[V]$, inductancia $L=12.6[\mathrm{mH}]$, resistencia $R=10.89[\Omega]$, condensador $C_{d c}=4.7[\mathrm{mF}]$, para el control de corriente de carga se estableció una corriente de referencia $i^{*}=50[A]$ a $50[\mathrm{~Hz}]$ de frecuencia.

\section{A. Respuesta en Estado Estacionario}

Las formas de onda de estado estacionario para corriente de carga trifásica, voltajes de condensadores del enlace DC y voltaje de salida del convertidor, utilizando un tiempo de muestreo de $T_{s}=100[\mu s]$ y $T_{s}=25[\mu s]$, se observan en las Figs. 6 y 7, respectivamente. Comparando ambas figuras, se observa que en ambos casos las corrientes de carga siguen a sus respectivas referencias, al mismo tiempo, los voltajes de los condensadores están muy bien equilibrados por los enfoques de control y la diferencia entre $v_{d c, u p}$ y $v_{d c, l o}$ es despreciable. Es posible observar que la ondulación o el error 
de la corriente disminuye al disminuir el tiempo de muestreo, y además se observa en el voltaje sobre la carga presenta una mejor onda sinusoidal cuando menor es el tiempo de muestreo.

a)

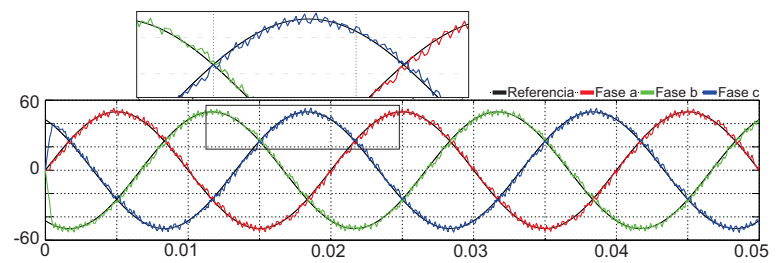

b)
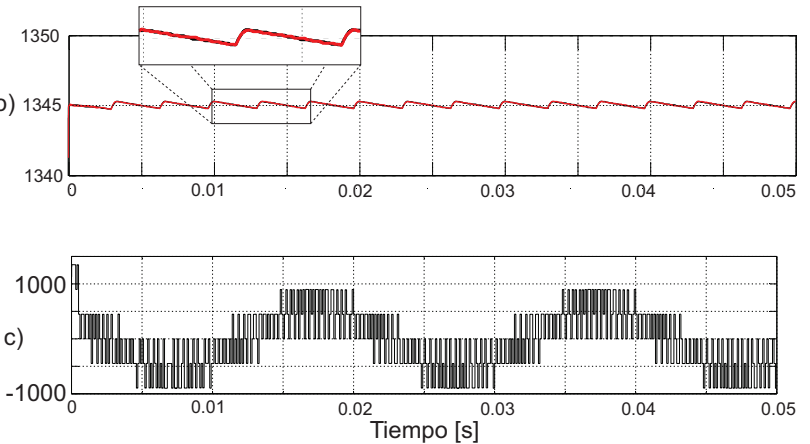

Fig. 6. Resultado de simulación de la técnica MPC aplicado al 3L-NPC para un tiempo de muestreo de $100 \mu \mathrm{s}$ : a) corriente de carga medida y corriente de referencia $[A], b)$ tensión en los capacitores del enlace DC $[V], c)$ tensión en la carga $[V]$

a)
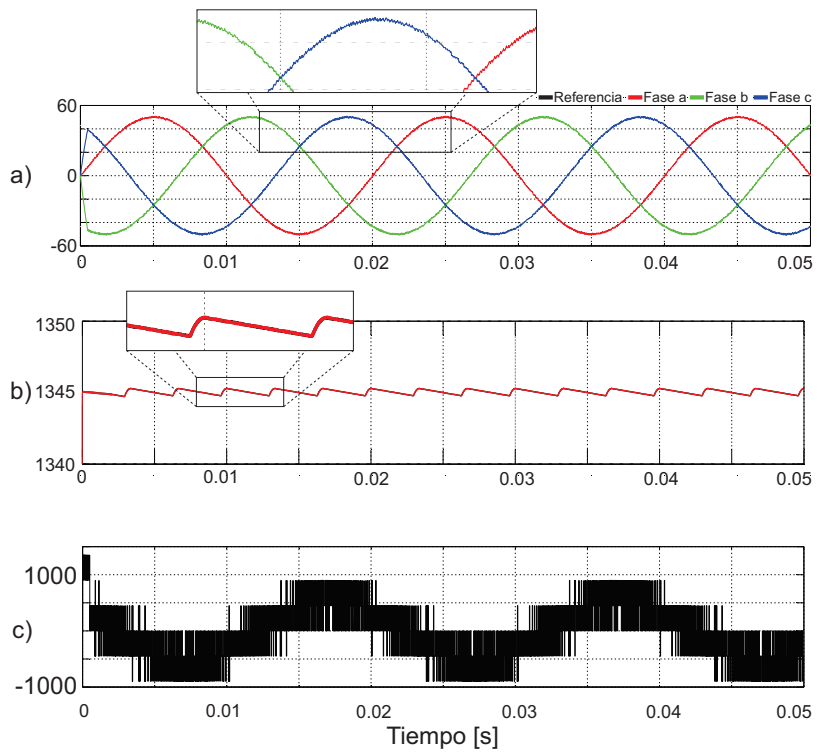

Fig. 7. Resultado de simulación de la técnica de control MPC aplicado al 3L-NPC para un tiempo de muestreo de $25 \mu \mathrm{s}$ : a) corriente de carga medida y corriente de referencia $[A], b)$ tensión en los capacitores del enlace DC $[V], c)$ tensión en la carga $[V]$.

\section{B. Respuesta Dinámica}

En la Fig. 8 se observa la simulación del MPC aplicado al 3L-NPC para un cambio de paso en la referencia. La corriente de referencia se hace variar en el instante $t=0.025[\mathrm{~s}] \mathrm{de}$ una magnitud igual a $i^{*}=50[A]$ pasa a $i^{*}=75[A]$. Se puede observar un buen comportamiento dinámico ya que los transitorios son suficientemente cortos. Además, se puede observar en la Fig. 8c una frecuencia de conmutación variable, donde la frecuencia de conmutación cambia en respuesta al cambio de paso en la corriente de carga.

a)


Fig. 8. Resultado de simulación de la técnica de control MPC aplicado al 3L-NPC para un cambio de referencia a un tiempo de muestreo de $25 \mu \mathrm{s}$ : a) corriente de carga medida y corriente de referencia $[A], b)$ tensión en los capacitores del enlace DC $[V], c)$ tensión en la carga $[V]$.

\section{CONCLUSIONES}

En este trabajo, se logró analizar el concepto de la técnica de control MPC aplicado a convertidores de potencia, destacando ventajas como, una buena respuesta dinámica, facilidad de incluir no linealidades, entre otras. Se logró la implementación del control MPC al 3L-NPC, para el control de la corriente a una carga trifásica, logrando balancear la tensión de los condensadores del enlace DC, y observar la respuesta de seguimiento del controlador al hacer variar el valor de la señal de referencia. Estos resultados obtenidos en las simulaciones demuestran que esta técnica de control es una alternativa viable para el control de convertidores de potencia, que funciona bien en condiciones constantes y transitorias, con un buen seguimiento de sus referencias y una ondulación reducida.

\section{AGRADECIMIENTOS}

Los autores agradecen el financiamiento del Proyecto de Investigación FONDECYT Regular 1191028, MEC 80190074, MEC 801800974 y FONDAP SERC Chile 15110019.

\section{REFERENCIAS}

[1] R. E. Pérez-Guzmán, M. Rivera, and P. W. Wheeler, "Recent advances of predictive control in power converters," in 2020 IEEE International Conference on Industrial Technology (ICIT), 2020, pp. 1100-1105.

[2] _ , "Predictive control strategies in power converters," in 2019 IEEE CHILEAN Conference on Electrical, Electronics Engineering, Information and Communication Technologies (CHILECON), 2019, pp. 1-6. 
[3] P. Zhou, K. Wang, and Y. Li, "An improved fcs-mpc algorithm for threephase five-level hybrid-clamed converter," in 2019 IEEE International Symposium on Predictive Control of Electrical Drives and Power Electronics (PRECEDE), 2019, pp. 1-5.

[4] J. Li, F. Yao, and H. Liu, "Harmonic analysis of current in dual-inverter using model predictive control strategy," in 2019 IEEE International Symposium on Predictive Control of Electrical Drives and Power Electronics (PRECEDE), 2019, pp. 1-4.

[5] R. E. Pérez-Guzmán, M. Rivera, J. A. Riveros, F. Herrera, and P. W. Wheeler, "Model predictive control applied to the three-phase neutral point clamped inverter," in 2020 IEEE International Conference on Industrial Technology (ICIT), 2020, pp. 493-498.

[6] S. Wodyk and G. Iwanski, "Control of three-phase power electronic converter with power controllers in stationary frame," IEEE Transactions on Industry Applications, vol. 56, no. 5, pp. 5257-5268, 2020.

[7] M. Majstorović, M. E. R. Abarca, and L. Ristic, "Review of mpc techniques for mmcs," in 2019 20th International Symposium on Power Electronics (Ee), 2019, pp. 1-7.

[8] R. E. Pérez-Guzmán, M. Rivera, N. Vicencio, and P. W. Wheeler, "Model-based predictive control in three-phase inverters," in 2020 IEEE International Conference on Industrial Technology (ICIT), 2020, pp. 499-504.

[9] M. Rivera, J. Rodriguez, and S. Vazquez, "Predictive control in power converters and electrical drives-part i," IEEE Transactions on Industrial Electronics, vol. 63, no. 6, pp. 3834-3836, 2016.
[10] F. Milano and A. Ortega Manjavacas, Power System Model, 2020, pp. $39-76$

[11] R. E. Pérez-Guzmán and M. Rivera, "Weighting factor selection in power converters based on model predictive control," in 2020 Congreso Estudiantil de Electrónica y Electricidad (INGELECTRA), 2020, pp. 1-

[12] Q. Wang, H. Yu, C. Li, X. Lang, S. S. Yeoh, T. Yang, M. Rivera, S. Bozhko, and P. Wheeler, "A low-complexity optimal switching timemodulated model-predictive control for pmsm with three-level npc converter,' IEEE Transactions on Transportation Electrification, vol. 6 , no. 3, pp. 1188-1198, 2020.

[13] Q. Wang, M. Rivera, J. A. Riveros, and P. Wheeler, "Modulated model predictive current control for pmsm operating with three-level npc inverter," in 2019 IEEE 15th Brazilian Power Electronics Conference and 5th IEEE Southern Power Electronics Conference (COBEP/SPEC), 2019, pp. 1-5.

[14] R. E. Pérez-Guzmán, F. Herrera, M. Rivera, J. Riveros, and P. W. Wheeler, "Implementation of predictive control in a three-phase neutral point clamped inverter," in 2019 IEEE CHILEAN Conference on Electrical, Electronics Engineering, Information and Communication Technologies (CHILECON), 2019, pp. 1-6.

[15] N. Bekhoucha, N. Mesbahi, and S. Ouchen, "Predictive current control of three level neutral point clamped grid connected inverter in photovoltaic generation systems," 102018. 